\title{
Klimawandel und die Chemie der Atmosphäre - wie wird sich die stratosphärische Ozonschicht entwickeln?
}

Martin Dameris*

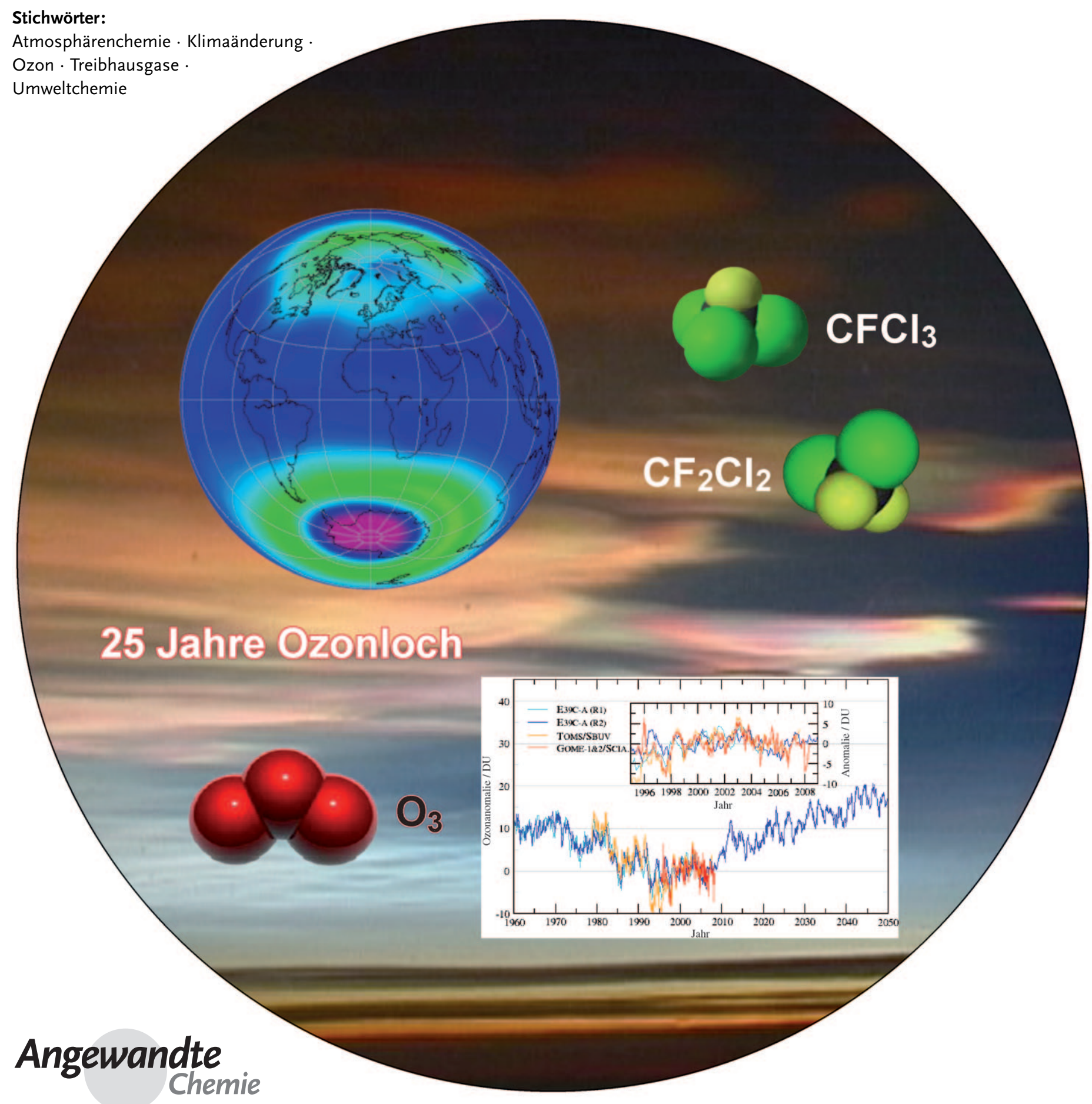


Die entdeckung des Ozonlochs über dem antarktischen Kontinent überraschte 1985 die Wissenschaft. Wenige Jahre wurde über die Ursachen für die Entstehung des Ozonlochs spekuliert. Bald wurde klar, dass durch die dort vorherrschenden meteorologischen Bedingungen eine besondere Situation entsteht: Sehr niedrige Temperaturen setzen chemische Prozesse in Gang, an deren Ende ein drastischer Abbau von Ozon in Höhen zwischen etwa 15 und $30 \mathrm{~km}$ steht. Dabei spielen so genannte polare Stratosphärenwolken eine Schlüsselrolle. Diese Wolken entstehen erst bei Temperaturen unterhalb von etwa $195 \mathrm{~K}$. Heterogene chemische Reaktionen auf den Wolkenpartikeln führen letztlich dazu, dass Ozonmoleküle zerstört werden. Die zukünftige Entwicklung der Ozonschicht hängt aber nicht nur davon ab, wie sich die Konzentrationen Ozon zerstörender Substanzen weiterentwickeln, sondern sie wird in hohem Maße auch vom Klimawandel beeinflusst werden.

\section{Einführung}

In den letzten Jahrzehnten haben zwei atmosphärische Veränderungen die Diskussion in der Wissenschaft und auch in der Öffentlichkeit geprägt: Der Klimawandel und das Ozonloch. Auf der einen Seite erhöhen sich seit Beginn der Industrialisierung vor etwa 150 Jahren die Konzentrationen von Treibhausgasen in der Erdatmosphäre mit entsprechenden Auswirkungen auf das Erdklima, auf der anderen Seite beobachtet man seit mehr als 25 Jahren eine massive Zerstörung der Ozonschicht, die durch die Emission von Fluorchlorkohlenwasserstoffen (FCKWs; z. B. FCKW-12 $=\mathrm{CF}_{2} \mathrm{Cl}_{2}$, $\mathrm{FCKW}-11=\mathrm{CFCl}_{3}, \mathrm{FCKW}-113=\mathrm{Cl}_{2} \mathrm{FC}-\mathrm{CClF}_{2}$ ) verursacht ist. In welcher Weise die bisherige und auch die zukünftige Entwicklung der Ozonschicht und des Klimas in Beziehung stehen, soll in diesem Aufsatz näher erläutert werden.

\subsection{Treibhauseffekt und Klimawandel}

Die Strahlung von der Sonne umfasst ein sehr breites Spektrum elektromagnetischer Wellen, von der harten Röntgenstrahlung (weniger als $0.1 \mathrm{~nm}$ Wellenlänge) bis hin zu langwelliger Radiostrahlung im Meterbereich. Am intensivsten ist die Solarstrahlung in den Wellenlängenbereichen der ultravioletten (UV-)Strahlung (etwa 100-380 nm Wellenlänge), des sichtbaren Lichts (etwa 380-780 nm) und des nahen Infrarots (bis zu etwa $3000 \mathrm{~nm}=3 \mu \mathrm{m}$ ). Das Maximum der Intensität befindet sich bei etwa $500 \mathrm{~nm}$.

Die solare Strahlung wird in der Atmosphäre teilweise reflektiert und absorbiert, oder sie durchdringt die Erdatmosphäre und erwärmt die Erde. Die erwärmte Erde emittiert ihrerseits Infrarotstrahlung (Wärmestrahlung) zurück in die Atmosphäre, wobei die Wellenlängen nun vor allem im Bereich des mittleren Infrarots (von 3 bis $50 \mu \mathrm{m}$ ) liegen (maximale Intensität bei etwa $10 \mu \mathrm{m}$ ). Der Unterschied zwischen der von der Sonne und der Erde ausgehenden Wärmestrahlung ergibt sich aufgrund der sehr unterschiedlichen Oberflächentemperaturen der Sonne (etwa $6000 \mathrm{~K}$ ) und der

\section{Aus dem Inhalt}

\begin{tabular}{lr}
\hline 1. Einführung & 3 \\
\hline 2. Chemie des stratosphärischen \\
Ozons \\
6 \\
\hline $\begin{array}{l}\text { 3. Dynamik der Stratosphäre und } \\
\text { Ozontransport }\end{array}$ \\
\hline $\begin{array}{l}\text { 4. Zukünftige Entwicklungen und } \\
\text { Konsequenzen } \\
\text { zum Schutionaler Abkommen der Atmosphäre }\end{array}$ \\
\hline \begin{tabular}{l} 
5. Zusammenfassung \\
\hline
\end{tabular}
\end{tabular}

Erde (etwa $288 \mathrm{~K})$. Treibhausgase, z. B. Kohlendioxid $\left(\mathrm{CO}_{2}\right)$, Methan $\left(\mathrm{CH}_{4}\right)$, Distickstoffoxid $\left(\mathrm{N}_{2} \mathrm{O}\right.$, bekannt auch als Lachgas), aber auch Wasserdampf $\left(\mathrm{H}_{2} \mathrm{O}\right)$ und Ozon $\left(\mathrm{O}_{3}\right)$, in der Erdatmosphäre verursachen nun deshalb eine Erwärmung der Troposphäre (atmosphärische Schicht, die sich vom Erdboden bis in etwa $12 \mathrm{~km}$ Höhe erstreckt), da die Infrarot (IR-)Strahlung, die von der Erde ausgesendet wird, durch die Treibhausgase absorbiert wird (Treibhauseffekt). Insgesamt hat dies zur Folge, dass sich nahe der Erdoberfläche im globalen Mittel eine Temperatur von etwa $288 \mathrm{~K}\left(+15^{\circ} \mathrm{C}\right)$ einstellt. Ohne diesen natürlichen Treibhauseffekt hätten die bodennahen Luftschichten im globalen Mittel lediglich eine Temperatur von etwa $255 \mathrm{~K}\left(-18^{\circ} \mathrm{C}\right)$.

Treibhausgase emittieren aber auch selbst IR-Strahlung, wobei die Emission von der lokalen Atmosphärentemperatur abhängt. In der Stratosphäre (Schicht in der Erdatmosphäre zwischen 12 und $50 \mathrm{~km}$ Höhe) emittieren Treibhausgase in der Regel mehr IR-Strahlung, als sie absorbieren, was eine Abkühlung der Stratosphäre zur Folge hat. Da die Temperatur in der Stratosphäre nach oben hin ansteigt (Effekt der Ozonschicht; siehe unten), nimmt die Emission von IRStrahlung mit der Höhe zu und ist im Bereich der höchsten Temperaturen, nahe der Stratopause in etwa $50 \mathrm{~km}$ Höhe, am größten. Dieser Abkühlungseffekt durch Treibhausgase variiert mit der geographischen Breite, da er vom Gleichgewicht zwischen der Absorption von IR-Strahlung (im Wesentlichen von unten) und lokaler Emission von IR-Strahlung abhängt.

Werden die Konzentrationen der Treibhausgase in der Erdatmosphäre nun verändert, so hat dies unmittelbaren Einfluss auf die Temperaturstruktur, da sich das Gleichge-

[*] Prof. Dr. M. Dameris

Deutsches Zentrum für Luft- und Raumfahrt Institut für Physik der Atmosphäre Oberpfaffenhofen, 82234 Weßling (Deutschland)

E-Mail: martin.dameris@dlr.de

Homepage: http://www.dlr.de/ipa 
wicht der Strahlungswirkung von der einfallenden solaren (kurzwelligen) Strahlung und der ausgehenden terrestrischen Strahlung (IR) in der Atmosphäre verändert. Wie bekannt, hat sich in den letzten 100 Jahren in Folge der stark gestiegenen Treibhausgaskonzentrationen die global gemittelte bodennahe Temperatur um $+0.74 \mathrm{~K}$ erhöht. ${ }^{[1]}$ Die Stratosphäre hingegen hat sich deutlich abgekühlt, was ebenfalls durch entsprechende Temperaturmessungen belegt ist. ${ }^{[2-4]}$ Sowohl die Analysen von Satelliten-gestützten Messungen als auch die von Ballon-getragenen Radiosonden zeigen eine sich mehr oder weniger stetig abkühlende Stratosphäre, wobei die ermittelten Trends wie erwartet eine Höhenabhängigkeit aufweisen (Abbildung 1): In der unteren Stratosphäre (ca. $16 \mathrm{~km}$ Höhe) findet man einen Abkühlungstrend von etwa $0.5 \mathrm{~K}$ pro Dekade; der Wert für die mittlere Stratosphäre (ca. $24 \mathrm{~km}$ Höhe) liegt bei etwa $1.2 \mathrm{~K}$ pro Dekade. ${ }^{[3]}$ Die Abkühlung der Stratosphäre in den 1980er und 1990er Jahren ist aber nicht nur durch die Zunahme der Treibhausgaskonzentrationen verursacht, sondern auch durch die Abnahme der stratosphärischen Ozonmenge, die den Abkühlungstrend vor allem in der unteren Stratosphäre maßgeblich beeinflusst hat. Modifiziert wird dieser negative stratosphärische Temperaturtrend durch eine Reihe natürlicher Vorgänge in der Atmosphäre, zum Beispiel den 11-jährigen Sonnenaktivitätszyklus oder starke Vulkanausbrüche. ${ }^{[5,6]}$

\subsection{Das Ozonloch}

Die größten Ozonmengen befinden sich in der Stratosphäre; etwa $90 \%$ des atmosphärischen Ozons befinden sich zwischen 15 und $30 \mathrm{~km}$ Höhe, in der so genannten Ozonschicht. In Äquatornähe findet man die höchsten Konzentrationen (etwa $5 \times 10^{12}$ Moleküle $\mathrm{cm}^{-3}$ ) in Höhen um etwa $25 \mathrm{~km}$, in höheren geographischen Breiten ist dieses Ozonmaximum nach unten verschoben (Abbildung 2). Der Grund dafür ist in der Dynamik der Stratosphäre und den damit verbundenen Ozontransporten zu suchen (siehe Abschnitt 3). Um die Gesamtmenge von Ozon in der Atmosphäre über einem bestimmten Ort anzugeben, nutzt man die so genannte Dobson-Einheit (Dobson Unit, DU; benannt nach Gordon

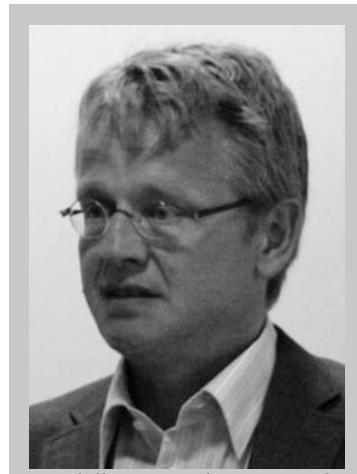

Martin Dameris ist Geophysiker und Meteorologe. Nach seinem Diplom im Jahre 1983 beendete er seine Dissertation 1987 an der Universität zu Köln. 1991 wechselte er zum Deutschen Zentrum für Luft- und Raumfahrt in Oberpfaffenhofen. Dort arbeitet er als Seniorwissenschaftler am Institut für Physik der Atmosphäre; er ist Professor am Meteorologischen Institut der Ludwig-Maximilians-Universität in München. Seine wissenschaftlichen Arbeiten konzentrieren sich auf die Untersuchung von Chemie-KlimaWechselwirkungen mittels eines numerischen Modellsystems der Troposphäre und der Stratosphäre. Er ist Autor und Koautor von mehr als 70 begutachteten wissenschaftlichen Arbeiten und er hat an einer Reihe internationaler Berichte zum Zustand der stratosphärischen Ozonschicht und des Klimas mitgewirkt.

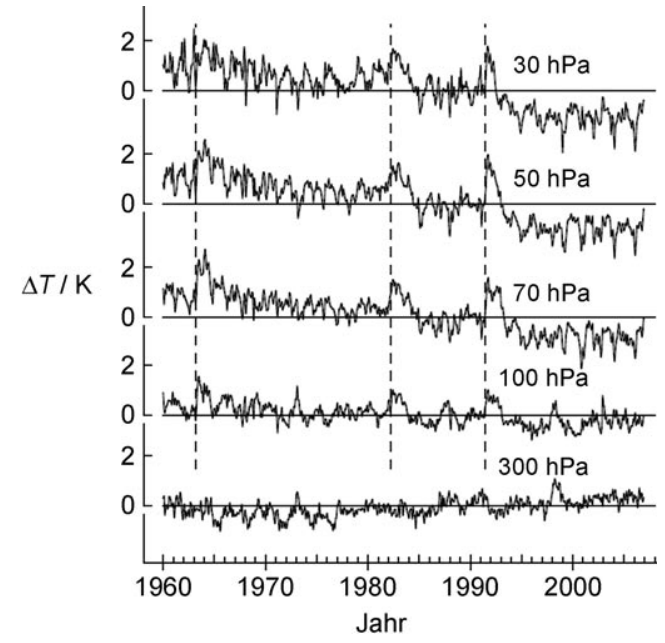

Abbildung 1. Zeitreihen global gemittelter Temperaturanomalien (bezogen auf das Langzeitmittel) auf verschiedenen atmosphärischen Druckniveaus, die von der oberen Troposphäre bis in die untere Stratosphäre reichen. Dabei entsprechen $300 \mathrm{hPa}$ einer Höhe von etwa $9 \mathrm{~km}$, $100 \mathrm{hPa}$ etwa $16 \mathrm{~km}, 70 \mathrm{hPa}$ etwa $18 \mathrm{~km}, 50 \mathrm{hPa}$ etwa $21 \mathrm{~km}$ und $30 \mathrm{hPa}$ etwa $24 \mathrm{~km}$. Die senkrechten gestrichelten Linien markieren Zeitpunkte großer Vulkanausbrüche: Agung (März 1963), El Chichon (April 1982) und Pinatubo (Juni 1991). Deutlich zu erkennen sind kurzzeitige Erhöhungen der stratosphärischen Temperatur (100-30 hPa) infolge der Vulkanausbrüche. Man erkennt einen negativen Temperaturtrend (Abkühlung) in den letzten 50 Jahren. Die Vulkanausbrüche wirken sich auf die Temperatur in der oberen Troposphäre (300 hPa) nicht so stark aus; der Temperaturtrend in den letzten Jahren ist hier leicht positiv (Erwärmung). (Abbildung 1 in [3].)

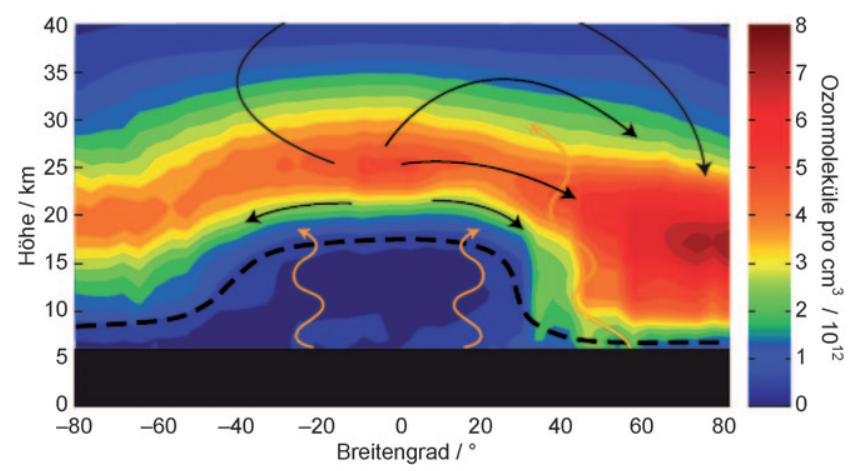

Abbildung 2. Skizze der Brewer-Dobson-Zirkulation und Darstellung der zonal gemittelten vertikalen Ozonverteilung im März 2004 (Messungen vom „Optical Spectrograph and InfraRed Imager System (OSIRIS) “ auf dem Odin-Satelliten). ${ }^{[32]}$ Die schwarzen Pfeile bezeichnen die stratosphärische Meridionalzirkulation, die Luftmassen aus tropischen Regionen in höhere Breiten in die untere Stratosphäre transportiert. Während der Wintermonate ist die Brewer-Dobson-Zirkulation verstärkt. Demzufolge wird im Frühling mehr Ozon in der Frühlingshemisphäre (in der Abbildung der rechte Teil) gemessen. Die Meridionalzirkulation wird durch atmosphärische Wellen, angedeutet durch orangefarbene Pfeile, angetrieben. Die gestrichelte schwarze Linie kennzeichnet den Übergangsbereich zwischen Troposphäre und Stratosphäre, die so genannte Tropopause. (Abbildung 1 in [33]. Copyright OSIRIS Science Team.) 
Dobson (1889-1976), der das erste Instrument zur Messung des atmosphärischen Ozonanteils konstruierte). DobsonEinheiten sind Säulendichten, also ein Maß für die Ozongesamtmenge in einer Säule über einen bestimmten Ort: Dabei entspricht eine $0.01 \mathrm{~mm}$ dicke Ozonschicht unter Normalbedingungen (1000 hPa, $273 \mathrm{~K})$ einer Dobson-Einheit (1 DU). Eine Ozonschichtdicke von 300 DU würde demnach an der Erdoberfläche einer reinen Ozonsäule von $3 \mathrm{~mm}$ entsprechen.

Die Ozonschicht in der Stratosphäre filtert einen großen Teil der von der Sonne ausgestrahlten UV-Strahlung (etwa 100-380 nm) und schützt somit das Leben auf der Erde. Besonders wichtig ist die nahezu vollständige Absorption der energiereichen solaren UV-B-Strahlung (Wellenlängenbereich zwischen 280 und $320 \mathrm{~nm}$ ). Durch die UV-B-Strahlung können vor allem Pflanzen, Tiere und der Mensch in Mitleidenschaft gezogen werden. So ist z. B. bekannt, dass erhöhte UV-B-Strahlung die Photosynthese beeinträchtigt, Hautkrebs hervorrufen kann und das Immunsystem schwächt. Auch das Leben in den oberen Regionen von Gewässern ist von der Intensität der UV-B-Strahlung direkt betroffen. Besonders empfindlich reagiert Phytoplankton. Die Absorption der solaren UV-Strahlung durch die stratosphärische Ozonschicht führt aber auch dazu, dass die Temperatur in der Stratosphäre mit der Höhe zunimmt und sich dadurch eine stabile Schichtung einstellt, die stärkere vertikale Luftbewegungen begrenzt. Dies spielt für das Klimasystem der Erde eine ganz wesentliche Rolle.

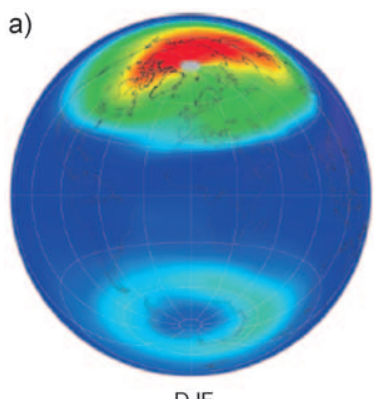

DJF

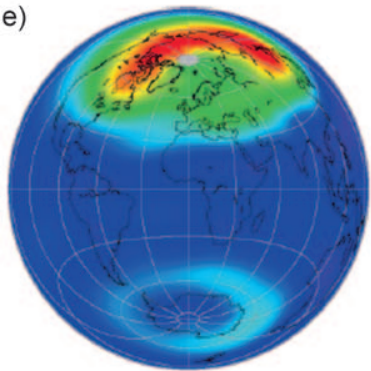

DJF

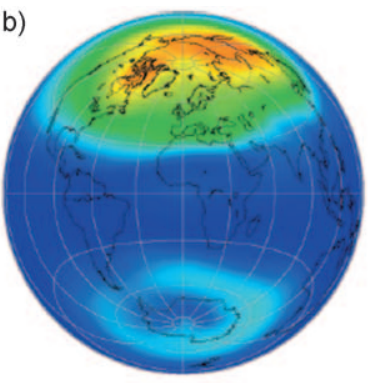

MAM f)

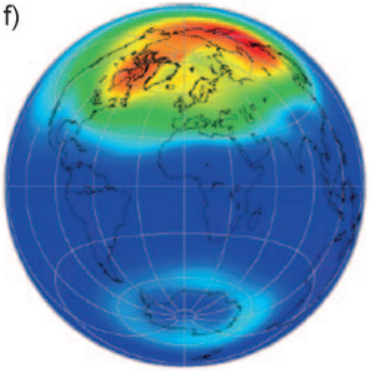

Gesamtozon / DU

200250300350400450

c)

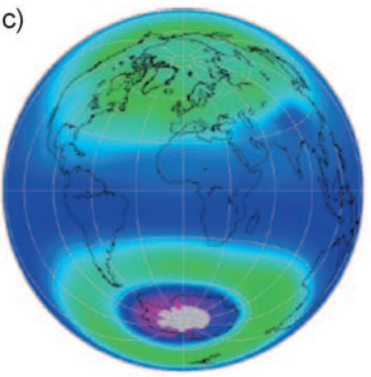

JJA

g)

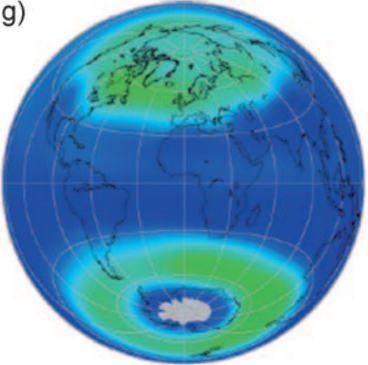

JJA

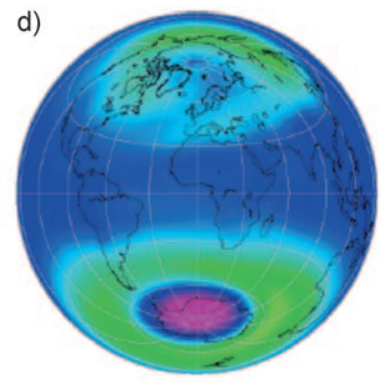

SON

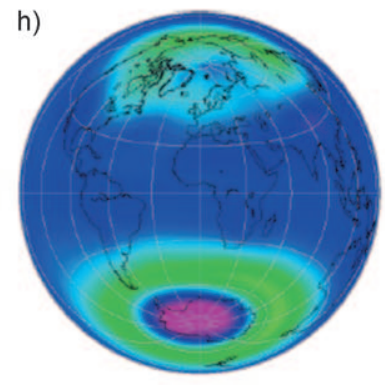

SON

250300350400450500

Abbildung 3. Über die Jahreszeiten gemittelte Gesamtozonwerte in Dobson-Einheiten (DU), abgeleitet aus a)-d) Satelliten-gestützten Messungen sowie e)-h) numerischen Simulationen mit dem Klima-Chemie-Modell E39C-A. Hierbei steht „DJF“ für Dezember, Januar, Februar, „MAM“ für März, April, Mai, „JJA“ für Juni, Juli, August und „SON“ für September, Oktober, November. Die dargestellten Mittelwerte repräsentieren den Zeitraum von Juni 1995 bis Mai 2008. (Abbildung 6 in [24].) 
vollständigen Abschaffung der Produktion und Verwendung von chlor- und bromhaltigen Chemikalien, die letztlich für die Zerstörung des stratosphärischen Ozons verantwortlich sind.

Die Dicke der Ozonschicht wird aber nicht nur durch chemische Prozesse in der Stratosphäre bestimmt - physikalische und dynamische Prozesse spielen eine ebenso wichtige Rolle. Ozon ist eines der wichtigsten strahlungsaktiven Gase in der Atmosphäre; es absorbiert kurz- und langwellige Strahlung und beeinflusst dadurch die vertikale Temperaturverteilung der Stratosphäre. Ferner wird Ozon als atmosphärisches Spurengas durch die stratosphärischen Windsysteme über große Distanzen transportiert. Dadurch wird die globale Ozonverteilung maßgeblich geprägt (siehe Abbildung 3). Ferner ist zu beachten, dass physikalische, dynamische und chemische Prozesse in der Atmosphäre zum Teil auf sehr komplexe Weise miteinander in Wechselwirkung stehen. Das Verständnis all dieser Prozesse und deren Wechselwirkungen ist wichtig, um einerseits kurzzeitige Fluktuationen der Ozonschichtdicke zu verstehen, andererseits aber auch langzeitliche Veränderungen (Trends) erklären zu können. Dieses Wissen ist notwendige Voraussetzung für belastbare Abschätzungen der zukünftigen Entwicklung der Ozonschicht. Veränderungen atmosphärischer Abläufe wegen des Klimawandels spielen hierbei eine nicht zu vernachlässigende Rolle.

Die Grundlagen und komplexen Zusammenhänge zur Bewertung bisheriger Veränderungen der Ozonschicht sowie zukünftig zu erwartender Entwicklungen sollen in diesem Aufsatz erläutert werden. In den folgenden Abschnitten werden die wichtigsten atmosphärischen Prozesse in der Atmosphäre beschrieben, die für die Entwicklung der stratosphärischen Ozonschicht von Bedeutung sind. Auf der Grundlage von Simulationen mithilfe numerischer Rechenmodelle werden Abschätzungen über die zukünftige Entwicklung der Ozonschicht vorgestellt sowie deren Unsicherheiten dargestellt und diskutiert.

\section{Chemie des stratosphärischen Ozons}

\subsection{Homogene Gasphasenchemie}

Atmosphärisches Ozon $\left(\mathrm{O}_{3}\right)$ wird ausschließlich photochemisch gebildet. Direkte Quellen von Ozon, z. B. am Erdboden, gibt es nicht. Die Bildung von Ozon in der Stratosphäre wird durch die Photolyse des molekularen Sauerstoffs $\left(\mathrm{O}_{2}\right)$ bei Wellenlängen unterhalb von etwa $240 \mathrm{~nm}$ durch die Reaktion gemäß Gleichung (1) initiiert. Hierbei entstehen zwei Sauerstoffatome im elektronischen Grundzustand $\left({ }^{3} \mathrm{P}\right)$, die im Folgenden durch Rekombination mit molekularem Sauerstoff Ozon bilden [mithilfe eines dritten Stoßpartners M, in der Regel Stickstoff oder Sauerstoff; Gl. (2)].

$\mathrm{O}_{2}+h v \rightarrow 2 \mathrm{O}\left({ }^{3} \mathrm{P}\right)$

$\mathrm{O}\left({ }^{3} \mathrm{P}\right)+\mathrm{O}_{2}+\mathrm{M} \rightarrow \mathrm{O}_{3}+\mathrm{M}$

Da Ozon photochemischen Ursprungs ist, ist seine Hauptbildungsregion die tropische und subtropische Strato- sphäre, in der die Sonne ganzjährig am intensivsten scheint. Gleichzeitig wird das so gebildete Ozon teilweise auch wieder abgebaut, zum einen durch die Photolyse des Ozons bei Wellenlängen unterhalb von etwa $300 \mathrm{~nm}$ und zum anderen durch die Reaktion mit einem Sauerstoffatom [Gl. (3) bzw. (4)]. Ob sich bei der Photolyse von Ozon (Reaktion 3) ein Sauerstoffatom im Grundzustand $\left({ }^{3} \mathrm{P}\right)$ oder im angeregten Zustand $\left({ }^{1} \mathrm{D}\right)$ bildet, hängt von der Wellenlänge ab. Die bisher genannten vier Reaktionen, die ausschließlich auf einer Sauerstoffchemie beruhen, werden nach ihrem Entdecker Sydney Chapman (1888-1970) auch als Chapman-Mechanismus bezeichnet ${ }^{[9]}$ und bilden die Basis für die Ozonchemie in der Stratosphäre.

$$
\begin{aligned}
& \mathrm{O}_{3}+h v \rightarrow \mathrm{O}\left({ }^{1} \mathrm{D},{ }^{3} \mathrm{P}\right)+\mathrm{O}_{2} \\
& \mathrm{O}_{3}+\mathrm{O} \rightarrow 2 \mathrm{O}_{2}
\end{aligned}
$$

Ermittelt man mithilfe dieses einfachen Reaktionssystems sowie der bekannten Geschwindigkeitskonstanten und Photolyseraten die stratosphärische Ozonmenge, so fällt diese im Vergleich mit gemessenen Werten um etwa einen Faktor $2 \mathrm{zu}$ hoch aus. Seit Anfang der 1950er Jahre ist bekannt, dass schnelle Katalysezyklen in Gegenwart des Katalysators X die Ozonmenge auf das beobachtete Maß reduzieren [Gl. $(5 \mathrm{a}, \mathrm{b})$ und (6)].

$$
\begin{aligned}
& \mathrm{X}+\mathrm{O}_{3} \rightarrow \mathrm{XO}+\mathrm{O}_{2} \\
& \mathrm{O}+\mathrm{XO} \rightarrow \mathrm{X}+\mathrm{O}_{2}
\end{aligned}
$$

Nettoreaktion: $\mathrm{O}+\mathrm{O}_{3} \rightarrow 2 \mathrm{O}_{2}$

Der Katalysator X wird beim Ozonabbau zu XO oxidiert (Reaktion 5a) und in der Folgereaktion (5b) mit einem Sauerstoffatom wieder zurückgebildet. Somit kann ein Katalysator diesen Reaktionszyklus bis zu einige Tausend Mal durchlaufen. Netto entspricht die oben genannte Sequenz [Reaktionen (5a,b)] der Reaktion (4), die aber durch die Gegenwart von X erheblich beschleunigt wird. Als Katalysatoren hat man bis Anfang der 1970er Jahre die Radikalpaare $(\mathrm{X} / \mathrm{XO}) \mathrm{OH} / \mathrm{HO}_{2}$ und $\mathrm{NO} / \mathrm{NO}_{2}$ identifiziert, die aus Wasserdampf $\left(\mathrm{H}_{2} \mathrm{O}\right)$ und Distickstoffmonoxid $\left(\mathrm{N}_{2} \mathrm{O}\right)$ gebildet werden. ${ }^{[10-12]}$ Mitte der 1970er Jahre wurden auch die Radikalpaare $\mathrm{Cl} / \mathrm{ClO}$ (aus FCKWs) sowie $\mathrm{Br} / \mathrm{BrO}$ (aus Halonen) als bedeutend ermittelt. ${ }^{[13,14]}$ Für ihre grundlegenden Arbeiten erhielten Paul J. Crutzen, Mario J. Molina und F. Sherwood Rowland 1995 den Nobelpreis für Chemie. ${ }^{[34]}$

Während der Wasserdampf natürlichen Ursprungs ist, hat Lachgas sowohl natürliche als auch anthropogene Quellen. FCKWs und Halone entstammen ausschließlich industrieller Produktion. Das natürliche stratosphärische Chlormischungsverhältnis liegt bei etwa $0.6 \mathrm{nmol} \mathrm{mol}^{-1}$; die wesentliche Quelle hierfür ist Chlormethan $\left(\mathrm{CH}_{3} \mathrm{Cl}\right)$, das in großen Mengen in Ozeanen und Böden produziert wird. Wegen der in den letzten Jahrzehnten in die Atmosphäre emittierten, großen Mengen an FCKWs hat man um die Jahrtausendwende stratosphärische Chlormischungsverhältnisse von deutlich über $3 \mathrm{nmol} \mathrm{mol}^{-1}$ gemessen. 
Das vermehrte Auftreten der FCKWs und Halone aufgrund anthropogener Emissionen hat deshalb den stratosphärischen Ozonabbauzyklus in den letzten Jahrzehnten deutlich verstärkt und den negativen Trend in der stratosphärischen Ozonmenge eingeleitet. FCKWs und Halone sind in der Troposphäre weitestgehend inert. Sie werden mit der Zeit (einige Jahre) in die Stratosphäre transportiert; erst dort werden sie photolysiert und in aktive Chlor- oder Bromverbindungen überführt. Gebremst wird die Wirkung der Radikale nur durch Reaktionen der Radikale untereinander [z. B. Gl. (7)-(9)]

$\mathrm{ClO}+\mathrm{NO}_{2}+\mathrm{M} \rightarrow \mathrm{ClONO}_{2}+\mathrm{M}$

$\mathrm{ClO}+\mathrm{HO}_{2} \rightarrow \mathrm{HOCl}+\mathrm{O}_{2}$

$\mathrm{Cl}+\mathrm{HO}_{2} \rightarrow \mathrm{HCl}+\mathrm{O}_{2}$

Dabei entstehen unter anderem Reservoirgase, z.B. Chlornitrat $\left(\mathrm{ClONO}_{2}\right)$ und Chlorwasserstoff $(\mathrm{HCl})$, die zwar einerseits das Ozonzerstörungspotential der Katalysatoren herabsetzen, andererseits aber auch wieder in die aktiven Formen zurückgeführt werden können. Ein ähnliches Schema lässt sich auch für Bromoxide $(\mathrm{Br}, \mathrm{BrO})$ ableiten.

Alle Quellgase der Ozon abbauenden Katalysatoren $\left(\mathrm{H}_{2} \mathrm{O}, \mathrm{N}_{2} \mathrm{O}, \mathrm{FCKW}\right.$, Halone) gelangen über die Troposphäre in die Stratosphäre. Das Freisetzen der jeweiligen Katalysatoren $\left(\mathrm{HO}_{x}, \mathrm{NO}_{x}, \mathrm{ClO}_{y}, \mathrm{BrO}_{y} ; x=1,2 ; y=0,1\right.$; abkürzende Schreibweise für die jeweiligen Radikalpaare) erfolgt im Wesentlichen photochemisch. Wegen der unterschiedlich starken Absorption solarer UV-Strahlung und auch der verfügbaren Solarstrahlung sind nicht alle Katalysatoren in allen Höhenbereichen der Stratosphäre gleichermaßen von Bedeutung, sondern es besteht eine Höhenabhängigkeit. Unter den Spezies, die den Ozonabbau bestimmen, ist $\mathrm{ClO}_{y}$ in der mittleren und unteren Stratosphäre (15 bis $30 \mathrm{~km}$ Höhe) dominant, dort wo sich am meisten Ozon befindet. In Höhen unterhalb von etwa $15 \mathrm{~km}$ bestimmt $\mathrm{HO}_{x}$ den Ozonabbau, oberhalb von etwa $30 \mathrm{~km}$ dominiert $\mathrm{NO}_{x}$ den Ozonabbau. Aus diesem Grund wird der beobachtete, negative Ozontrend in den letzten Jahrzehnten vor allem auf das anthropogene $\mathrm{ClO}_{y}$ zurückgeführt.

\subsection{Heterogene chemische Prozesse}

Wie in niedrigen und mittleren geographischen Breiten kann Ozon auch im polaren Winter durch $\mathrm{HO}_{x^{-}}, \mathrm{NO}_{x^{-}}, \mathrm{ClO}_{y^{-}}$ und $\mathrm{BrO}_{y}$-katalysierte Zyklen abgebaut werden. Allerdings sind diese Prozesse sehr langsam, weil unter den dortigen Zwielichtbedingungen nur wenig UV-Strahlung vorhanden ist. In der polaren Stratosphäre sind es vor allem chemische Reaktionen auf den Oberflächen von polaren stratosphärischen Eisteilchen, die für eine Aktivierung des Chlors (oder Broms) in der Stratosphäre verantwortlich sind und das so aktivierte Chlor zur beobachteten Ozonzerstörung unmittelbar nach Ende der Polarnacht führt. ${ }^{[15]}$

In der sehr kalten polaren unteren Stratosphäre bilden sich während der Wintermonate in der Polarnacht Strato- sphärenwolken (Polar Stratospheric Clouds, PSCs; Abbildung 4). Bei typischen Wasserdampf- $\left(4.5 \mathrm{mmol} \mathrm{mol}^{-1}\right.$ in etwa $20 \mathrm{~km}$ Höhe) oder Salpetersäure-Mischungsverhältnissen $\left(10 \mathrm{nmol} \mathrm{mol}^{-1}\right.$ in etwa $20 \mathrm{~km}$ Höhe) entstehen PSCs bei

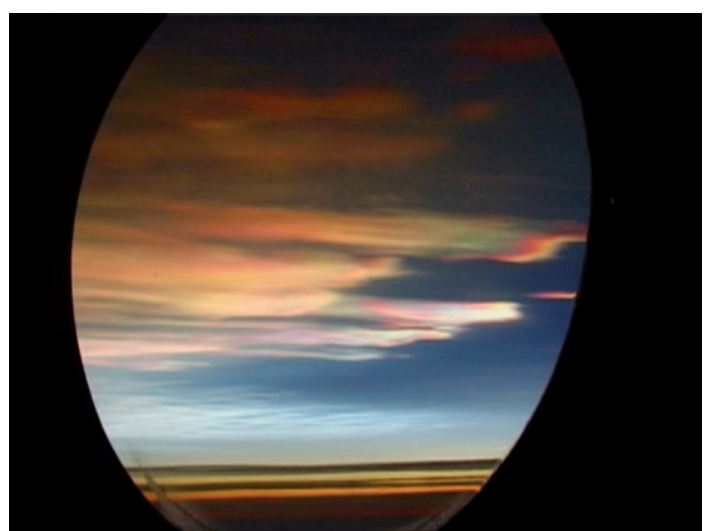

Abbildung 4. Polare Stratosphärenwolken über Finnland. Die Aufnahme wurde am 26. Januar 2000 aus dem DLR-Forschungsflugzeug Falcon aufgenommen.

Temperaturen unterhalb von etwa $195 \mathrm{~K}\left(-78^{\circ} \mathrm{C}\right)$. Es entstehen Salpetersäure-Trihydrat-Kristalle $\left(\mathrm{HNO}_{3} \times 3 \mathrm{H}_{2} \mathrm{O}=\right.$ Nitric Acid Trihydrate $=$ NAT). Unter den genannten typischen Bedingungen in der unteren Stratosphäre hat NAT eine um etwa 7-8 K höhere Gleichgewichtstemperatur als Eis (Eis entwickelt sich unterhalb von etwa $\left.188 \mathrm{~K}\left(-85^{\circ} \mathrm{C}\right)\right)$, entsteht also beim Abkühlen deutlich früher und häufiger. Aus Gründen der unterschiedlichen Land-See-Verteilung auf der Nord- und Südhalbkugel kühlt sich die untere Stratosphäre über dem Südpol im Winter (Juni-August) deutlich stärker ab als die nordpolare Stratosphäre (Dezember-Februar). Im klimatologischen Mittel liegen die polaren Wintertemperaturen in der unteren arktischen Stratosphäre um etwa $10 \mathrm{~K}$ höher als in der unteren antarktischen Stratosphäre. Während die antarktische Stratosphäre in jedem Jahr einige Wochen lang auf Temperaturen unterhalb der PSC-Bildungstemperaturen sinkt, beobachtet man in der nordpolaren Stratosphäre eine stark ausgeprägte Jahr-zu-Jahr-Variabilität: Es gibt einerseits relativ warme Winter, in denen kaum PSCs entstehen, andererseits aber auch sehr kalte Winter mit Antarktis-ähnlichen Bedingungen. Dies führt dazu, dass sich in der antarktischen Stratosphäre in jedem Winter sehr ausgedehnte PSC-Felder entwickeln, die man so über der Arktis nur ganz selten beobachtet.

Zum einen ermöglichen PSC-Teilchen auf ihren Oberflächen heterogene Reaktionen, durch die Halogenverbindungen aus Reservoirverbindungen freigesetzt und letztlich in eine aktivere Form überführt werden können. Die wichtigsten heterogenen Reaktionen sind in den Gleichungen (10)-(14) genannt. Beim Wiedererscheinen der Sonne im polaren Frühling werden aktive Moleküle wie $\mathrm{Cl}_{2}$ oder $\mathrm{HOCl}$ in reaktives $\mathrm{Cl}$ bzw. $\mathrm{ClO}$ überführt; die Ozonzerstörung beginnt [Reaktionen (5) und (6)].

$\mathrm{ClONO}_{2}(\mathrm{~g})+\mathrm{HCl}(\mathrm{s}) \rightarrow \mathrm{HNO}_{3}(\mathrm{~s})+\mathrm{Cl}_{2}(\mathrm{~g})$ 
$\mathrm{ClONO}_{2}(\mathrm{~g})+\mathrm{H}_{2} \mathrm{O}(\mathrm{s}) \rightarrow \mathrm{HNO}_{3}(\mathrm{~s})+\mathrm{HOCl}(\mathrm{g})$

$\mathrm{HOCl}(\mathrm{g})+\mathrm{HCl}(\mathrm{s}) \rightarrow \mathrm{Cl}_{2}(\mathrm{~g})+\mathrm{H}_{2} \mathrm{O}(\mathrm{s})$

$\mathrm{N}_{2} \mathrm{O}_{5}(\mathrm{~g})+\mathrm{H}_{2} \mathrm{O}(\mathrm{s}) \rightarrow 2 \mathrm{HNO}_{3}(\mathrm{~s})$

$\mathrm{N}_{2} \mathrm{O}_{5}(\mathrm{~g})+\mathrm{HCl}(\mathrm{s}) \rightarrow \mathrm{ClNO}_{2}(\mathrm{~g})+\mathrm{HNO}_{3}(\mathrm{~s})$.

Zum Anderen können PSC-Partikel wegen ihrer Größe und ihres Gewichts sedimentieren und dadurch Stickstoffverbindungen in der Stratosphäre in tiefer liegende Schichten verfrachten („Denitrifizierung“). Damit wird die Möglichkeit vermindert, dass nach dem Schmelzen der PSC-Teilchen lokal die schädlichen Chlorverbindungen wieder schnell desaktiviert werden. Die Freisetzung und die Photolyse von gasförmigem $\mathrm{HNO}_{3}$ wird in bestimmten Höhen vermindert und die Bildung von neuem $\mathrm{NO}_{2}$ reduziert, das durch eine Rekombinationsreaktion [Gl. (15)] zur Desaktivierung der Chloroxid-Radikale beiträgt. Der $\mathrm{NO}_{x}$-Zyklus verliert damit an Bedeutung, und der $\mathrm{ClO}_{y}$-Zyklus wird durch das freigesetzte Chlor beschleunigt. In der polaren unteren Stratosphäre wird die Effizienz des $\mathrm{ClO}_{y}$-Zyklus aber vor allem dadurch gesteigert, dass er wegen der sehr geringen Zahl an Sauerstoffatomen bei Zwielichtbedingungen nicht gemäß Gleichung (16), abläuft, sondern über die Bildung von $\mathrm{ClOOCl}$ [Gl. (17)-(19)]. Das ClO-Dimer $\mathrm{ClOOCl}$ wird selbst bei tief stehender Sonne leicht photolysiert und setzt Cl-Atome frei, die Ozon abbauen. ${ }^{[16]}$ Damit hat die Photolyse der ClODimere [Gl. (18)] eine Schlüsselrolle bei der Ozonzerstörung in polaren Bereichen. Der Vollständigkeit halber sei noch gesagt, dass neben der Reaktion (15) auch die Reaktion (20) eine sehr wichtige Rolle bei der Desaktivierung von reaktivem Chlor spielt.

$$
\begin{aligned}
& \mathrm{ClO}+\mathrm{NO}_{2}+\mathrm{M} \rightarrow \mathrm{ClONO}_{2}+\mathrm{M} \\
& \mathrm{ClO}+\mathrm{O} \rightarrow \mathrm{Cl}+\mathrm{O}_{2} \\
& \mathrm{ClO}+\mathrm{ClO}+\mathrm{M} \rightarrow \mathrm{ClOOCl}+\mathrm{M} \\
& \mathrm{ClOOCl}+h v \rightarrow \mathrm{Cl}+\mathrm{ClOO} \\
& \mathrm{ClOO}+\mathrm{M} \rightarrow \mathrm{Cl}+\mathrm{O}_{2}+\mathrm{M} \\
& \mathrm{Cl}+\mathrm{CH}_{4} \rightarrow \mathrm{HCl}+\mathrm{CH}_{3}
\end{aligned}
$$

An dieser Stelle sei kurz erwähnt, dass bei eruptiven, sehr starken Vulkanausbrüchen große Mengen an Staubteilchen und chemischen Substanzen direkt in die Stratosphäre injiziert werden. So wurden z. B. bei dem Ausbruch des Pinatubo auf den Philippinen im Juni 1991 nahezu 20 Millionen Tonnen Schwefeldioxid in die Stratosphäre eingetragen. Das $\mathrm{SO}_{2}$ wurde in der Stratosphäre oxidiert, und es bildeten sich Schwefelsäuretröpfchen. Diese haben in der Stratosphäre eine sehr ähnliche Wirkung wie PSCs, jedoch mit dem Unterschied, dass die heterogenen chemischen Reaktionen bereits bei höheren Temperaturen beginnen. Die Wirkung der Vulkane auf die Ozonschicht im Speziellen und auf das Klima im Allgemeinen ist allerdings auf einige wenige Jahre beschränkt.
Zum Ende dieses Abschnittes sei noch darauf verwiesen, dass eine Vielzahl der chemischen Reaktionen in der Atmosphäre von der jeweils vorherrschenden Temperatur abhängt. Dies sei kurz an folgendem für die Ozonchemie wichtigen Beispiel erläutert:

Die wichtigsten Ozonabbauzyklen im Bereich der mittleren und oberen Stratosphäre (verursacht durch die Katalysatoren $\mathrm{HO}_{x}, \mathrm{NO}_{x}, \mathrm{ClO}_{y}$ und $\mathrm{BrO}_{y}$ ) verlangsamen sich bei abnehmender Temperatur, was höhere Ozonkonzentrationen nach sich zieht. ${ }^{[17]}$ In der Mesosphäre (Atmosphärenschicht oberhalb von etwa $50 \mathrm{~km}$ bis etwa $100 \mathrm{~km}$, in der es auch Ozonmoleküle gibt) hängt der Anstieg der Ozonkonzentration wesentlich $a b$ von der negativen Temperaturabhängigkeit der Reaktion (2). Komplizierter stellt sich die Situation im Bereich der oberen Stratosphäre dar, wo die verschiedenen Ozonabbauzyklen größeren Einfluss auf die Ozonmengen haben und dies zudem unterschiedlich in verschiedenen Höhen (z. B. $\mathrm{HO}_{x}$ im Bereich 50-60 km, $\mathrm{NO}_{x}$ unterhalb von etwa $45 \mathrm{~km}$ ). Dort werden die langsameren Ozonverluste nicht nur durch die Temperaturabhängigkeit der Reaktionsgeschwindigkeiten kontrolliert, sondern auch durch die Verringerung der Mengen von atomarem Sauerstoff. Die alle Ozonverlustzyklen limitierenden Geschwindigkeitskonstanten sind proportional der Anzahldichte von atomarem Sauerstoff, die ihrerseits stark durch die Reaktion (2) bestimmt wird. Insgesamt nimmt so der Ozongehalt in der mittleren und oberen Stratosphäre bei sinkenden Temperaturen zu, da sich die wichtigen Ozon abbauenden Reaktionen (homogene Gasphasenchemie) verlangsamen. Dagegen führen niedrigere Temperaturen in der polaren unteren Stratosphäre zur verstärkten Bildung von PSCs und somit potenziell zu einem stärkeren Ozonabbau durch heterogene chemische Reaktionen.

Die bisher dargestellten chemischen Abläufe in der Stratosphäre beschreiben lediglich die allerwichtigsten, grundlegenden chemischen Prozesse, die gerade für die Ozonchemie relevant sind. Sie reichen soweit aus, um die wesentlichen Abläufe weitestgehend richtig zu beschreiben. Heute verwendete numerische Modelle, die eine ausführliche Beschreibung der stratosphärischen Chemie enthalten, berücksichtigen allerdings mehr als 30 chemische Spezies, deren Konzentrationen in mehreren 100 chemischen Reaktionen eingehen. Dies soll hier aber nicht näher erläutert werden.

\section{Dynamik der Stratosphäre und Ozontransport}

Atmosphärische Spurengaskonzentrationen werden nicht nur durch chemische Prozesse verändert, sondern können auch durch Luftmassentransporte beeinflusst werden, die durch die vorherrschenden Windfelder (Windstärke und -richtung) bestimmt werden. Im welchem Ausmaß ein solcher Transport von Spurengasen stattfindet, hängt von der Lebensdauer der jeweiligen chemischen Spezies ab. Ozon in der oberen und mittleren Stratosphäre ist z. B. recht kurzlebig, da seine Abbaureaktionen durch Photolyse und mit Sauerstoffatomen [Gl. (3) und (4)] relativ schnell ablaufen. Aus diesem Grund spielt der Transport von Ozon in diesen Höhenbereichen nur eine untergeordnete Rolle. In der unteren Strato- 
sphäre hingegen ist die chemische Lebensdauer von Ozon deutlich höher, weshalb Transportprozesse hier eine wichtige Rolle im Hinblick auf die geographische Verteilung spielen. Ozon kann in diesen Höhen somit auch in geographische Breiten transportiert werden, in denen es photochemisch kaum entsteht. So wird z. B. das in den niedrigen (tropischen) und mittleren Breiten gebildete Ozon wegen der großräumigen Meridionalzirkulation (siehe weiter unten in diesem Abschnitt) in der Stratosphäre besonders effektiv in Richtung des jeweiligen Winterpols transportiert (d.h. von Dezember bis Februar in Richtung Nordpolarregion, von Juni bis August in Richtung Südpolarregion) und in die dortigen Luftmassen eingemischt. Dies führt dazu, dass die globale Ozonverteilung eine Asymmetrie mit maximalen Werten in den höheren geographischen Breiten, während der jeweiligen Frühlingsmonate, und nicht über dem Äquator, aufweist. In den tropischen Bereichen werden hingegen relativ geringe Ozonmengen gefunden (Abbildung 5). Somit ist es vor allem in höheren geographischen Breiten der Stratosphäre schwierig, die chemischen Einflüsse auf die Ozonverteilung (Ozonverlustraten) von den durch dynamische Prozesse verursachten Veränderungen zu trennen.

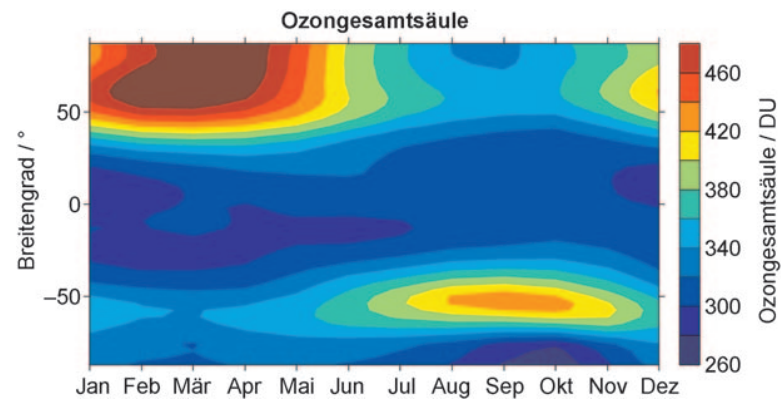

Abbildung 5. Gemittelte globale Verteilung der atmosphärischen Ozongesamtsäule simuliert mit dem gekoppelten Klima-Chemie-Modell E39C-A. Die Konturlinien gleicher Ozonmengen sind in Dobson-Einheiten dargestellt.

Die stratosphärische Meridionalzirkulation (nach den beiden Wissenschaftlern Alan Brewer (1915-2007) und Gordon Dobson (1889-1976) in Anerkennung ihrer grundlegenden Arbeiten zur Untersuchung von atmosphärischem Wasserdampf und Ozon auch Brewer-Dobson-Zirkulation genannt $)^{[18,19]}$ ist der bedeutendste Antrieb für den Transport von stratosphärischen Luftmassen aus niederen in höhere geographische Breiten. Ihre Klimatologie wird gekennzeichnet durch aufsteigende Luftmassen aus der Troposphäre in den Tropen in die Stratosphäre und dort durch einen polwärts gerichteten (in den Wintermonaten verstärkten) Transport (Abbildung 2). Aufgrund der Massenerhaltung beobachtet man in höheren Breiten ein Absinken von stratosphärischen Luftmassen, was unter anderem dazu führt, dass stratosphärische Luft zurück in die Troposphäre verfrachtet wird.

Auch bezüglich der atmosphärischen Zirkulation wird erwartet, dass der Klimawandel die Prozesse modifiziert, welche die Zirkulationssysteme antreiben. Dies hätte zur Folge, dass sich sowohl die Intensität der Luftmassentransporte als auch die Transportwege ändern, mit möglicherweise nachhaltigen Konsequenzen für die atmosphärische Verteilung von klimarelevanten Spurengasen, zu denen auch Ozon gehört. Dies wiederum kann zu rückkoppelnden Effekten auf das Erdklima führen.

Obwohl die dynamischen Prozesse, welche die stratosphärische Zirkulation steuern, recht gut verstanden sind, gibt es derzeit noch große Unsicherheiten hinsichtlich der Veränderlichkeit dieser Prozesse und ihrer Wirkung in einem sich wandelnden Klima (siehe Abschnitt 4). Bisherige Analysen von Beobachtungsdaten liefern noch keine eindeutigen und schlüssigen Hinweise auf eine durch den Klimawandel geänderte stratosphärische Zirkulation. Eine Intensivierung der meridionalen Zirkulation hätte z.B. zur Folge, dass sich die außertropische Atmosphäre erwärmt (adiabatisches Absinken) und die tropische Atmosphäre abkühlt (adiabatisches Aufsteigen). Dies hätte direkte Auswirkungen für den Eintrag von troposphärischen Quellgasen in die Stratosphäre (also solchen, die ursprünglich in der Troposphäre emittiert werden, in die Stratosphäre aufsteigen und dort chemisch umgewandelt werden), von so genannten Produktgasen (Zwischen- oder Endprodukte, die in der Troposphäre erzeugt werden) und auch von Wasserdampf, der in der Troposphäre viel höhere Konzentrationen als in der Stratosphäre aufweist (die Wasserdampfkonzentration in Erdbodennähe und die in etwa $15 \mathrm{~km}$ Höhe unterscheiden sich um etwa 4 Größenordnungen); aber es könnten auch verstärkt stratosphärische Luftmassen mit hohen Ozonkonzentrationen (oder geringen Wasserdampfkonzentrationen) in die Troposphäre transportiert werden, wo die Ozonkonzentrationen (Wasserdampfkonzentrationen) im Normalfall deutlich geringer (höher) sind. Diese Umverteilungen würden wiederum auf den Strahlungshaushalt der Atmosphäre und auch auf chemische Prozesse rückkoppeln.

\section{Zukünftige Entwicklungen und Konsequenzen internationaler Abkommen zum Schutz der Atmosphäre}

Als Folge der internationalen Vereinbarungen zum Schutz der stratosphärischen Ozonschicht (Montreal-Protokoll) konnte der rasche Konzentrationsanstieg der wichtigsten FCKWs (FCKW-11, -12, -113) in der Troposphäre gestoppt werden (Abbildung 6). Bereits seit Mitte der 1990er Jahre beobachtet man einen Rückgang des troposphärischen FCKW-Gehalts. Infolgedessen beobachtet man nun seit einigen Jahren auch einen leichten Rückgang der Chlorkonzentration in der Stratosphäre. Wegen der langen Lebenszeiten der FCKWs in der Atmosphäre wird es aber noch etwa bis Mitte dieses Jahrhunderts dauern, bis der Chlorgehalt der Stratosphäre wieder auf Werte zurückgeht, die noch in den 1960er Jahren vorherrschten. Aus diesem Grund geht man davon aus, dass sich der in den letzten drei Jahrzehnten beobachtete, starke chemische Ozonabbau in nächster Zeit verringern wird; die Dicke der Ozonschicht sollte allmählich wieder zunehmen, und das Ozonloch über der Antarktis sollte sich wieder schließen. ${ }^{[20]}$ Die Geschwindigkeit der Rückbildung der Ozonschicht und deren weitere, zukünftige Entwicklung sind jedoch noch von einer Reihe anderer Fak- 

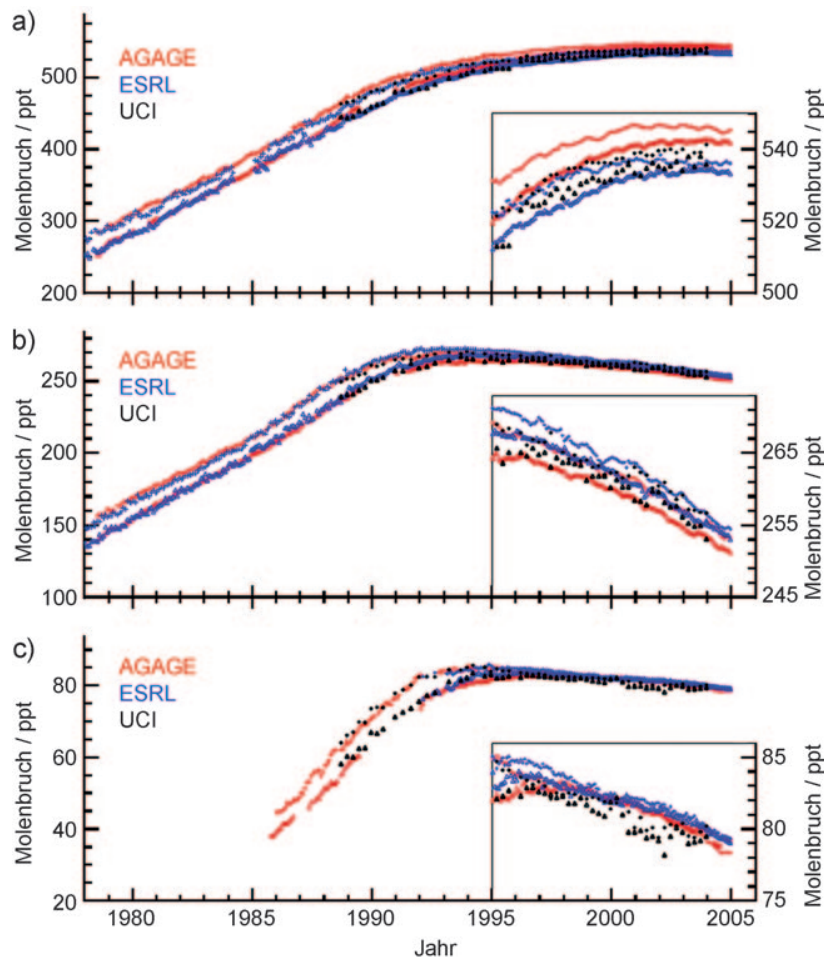

Abbildung 6. Hemisphärische Monatsmittelwerte der troposphärischen Mischungsverhältnisse (in ppt $=$ parts per trillion $=10^{-12}$ ) der wichtigsten FCKWs: a) CFC-12= $\mathrm{CF}_{2} \mathrm{Cl}_{2}$, b) CFC-11 = $\mathrm{CFCl}_{3}$ und c) CFC$113=\mathrm{Cl}_{2} \mathrm{FC}-\mathrm{CClF}_{2}$. Kreuze symbolisieren Messwerte für die Nordhemisphäre, Dreiecke solche für die Südhemisphäre. Jüngste Messwerte sind in den Einschüben vergrößert dargestellt. AGAGE = Advanced Global Atmospheric Gases Experiment; ESRL = Earth System Research Laboratory; $\mathrm{UCl}=$ University of California, Irvine. (Abbildung 1-1 in [20].)

toren abhängig: Steigende atmosphärische Konzentrationen von strahlungsaktiven Gasen (unter anderem $\mathrm{CO}_{2}, \mathrm{CH}_{4}$, $\mathrm{N}_{2} \mathrm{O}$ ) führen nicht nur dazu, dass sich die atmosphärischen Bedingungen in der Troposphäre verändern (Stichwort Treibhauseffekt), sondern auch die in der Stratosphäre (siehe Abschnitte 2 und 3). Die Rückbildung der Ozonschicht geschieht also unter anderen atmosphärischen Rahmenbedingungen als der Prozess der Ozonzerstörung in den vergangenen Jahrzehnten. Bedingt durch den Klimawandel ist es also sehr unwahrscheinlich, dass sich die Ozonschicht exakt wieder hin zu einem Zustand entwickeln wird, der jenem in den Zeiten vor erhöhten Konzentrationen Ozon zerstörender Substanzen entspricht.

Wie bereits erwähnt, bestimmen außer chemischen Prozessen auch dynamische und physikalische Prozesse die Struktur der Ozonschicht. Weil all diese Prozesse auf recht komplexe Art und Weise miteinander im Wechsel wirken, ist die Abschätzung der zukünftigen Entwicklung der stratosphärischen Ozonschicht wissenschaftlich äußerst anspruchsvoll. Deshalb weichen die verfügbaren Abschätzungen, die meistens auf Ergebnissen numerischer Atmosphärenmodelle beruhen, zum Teil noch deutlich voneinander ab. Auf der Grundlage des derzeitigen Verständnisses atmosphärischer Prozesse sowie zu erwartender Veränderungen durch den
Klimawandel können aber hinsichtlich der weiteren Entwicklung der stratosphärischen Ozonschicht trotzdem einige belastbare Aussagen getroffen werden.

\subsection{Weitere Entwicklung der Ozonschicht}

In den kommenden Jahrzehnten werden sich wegen der weiter steigenden Treibhausgaskonzentrationen die Atmosphärentemperaturen global weiter verändern, d.h., für die Stratosphäre wird erwartet, dass sie sich wegen der Strahlungseffekte weiter abkühlt. Genaue Angaben über die Stärke des zu erwartenden Trends sind schwierig, da die zukünftigen Treibhausgasemissionen unsicher sind. ${ }^{[1]}$ Zusätzlich berücksichtigt werden muss aber auch, dass sich wegen der zu erwartenden Rückbildung der Ozonschicht die stratosphärischen Ozonheizraten (Absorption solarer UV-Strahlung durch Ozon) wieder erhöhen werden, was der verstärkten Abkühlung durch erhöhte Treibhausgaskonzentrationen zum Teil entgegenwirkt. Da die Ozonkonzentration ihrerseits aber wiederum sehr stark von der jeweiligen Hintergrundtemperatur abhängt, gibt es hier eine Rückkopplung. In Anbetracht der Tatsache, dass sich bei einem Klimawandel auch die Dynamik der Stratosphäre verändern wird, kann es je nach Jahreszeit und Ort durchaus auch zu „dynamischen“ Erwärmungen der Stratosphäre kommen, sodass sich die Stratosphäre lokal erwärmt und nicht abkühlt. Dies gilt in besonderem Maße für die nordpolare Stratosphäre in den Winterund Frühlingsmonaten. ${ }^{[21]}$ Deshalb ist es sowohl für die Interpretation beobachteter Veränderungen der Ozonschicht als auch für prognostische Studien erforderlich, die Kopplung von chemischen, physikalischen und dynamischen Prozessen zu berücksichtigen. Es ist offensichtlich, dass Abschätzungen der zukünftigen Entwicklung der stratosphärischen Ozonkonzentration nicht trivial und deshalb auch mit Unsicherheiten behaftet sind. Die engen Verbindungen der Änderung der chemischen Zusammensetzung der Atmosphäre (Stichwort Montreal-Protokoll) einerseits und des Klimas (Stichwort Kyoto-Protokoll) andererseits spielen dabei eine sehr wichtige Rolle. Wegen der genannten Zusammenhänge könnte eine weitere Abkühlung der polaren unteren Stratosphäre zu einer Verzögerung bei der Rückbildung des antarktischen Ozonlochs führen, auf der anderen Seite könnte sich in anderen Bereichen der Stratosphäre die Rückbildung der Ozonschicht beschleunigen.

Veränderungen des Klimas und der Ozonschicht können mithilfe von Klima- und gekoppelten Klima-Chemie-Modellen (Chemistry-Climate Models, CCMs) nachvollzogen werden. Dabei handelt es sich um numerische Rechenmodelle mit deren Hilfe physikalische, dynamische und chemische Prozesse sowie deren Wechselwirkungen simuliert werden. ${ }^{[22]}$ Bei solchen numerischen Studien ist es erforderlich, dass außer natürlichen Vorgängen und ihren Variationen auch menschliche Eingriffe berücksichtigt werden, die für die Beschreibung atmosphärischer Vorgänge relevant sind. Besonders zu erwähnen sind hierbei die Variation der Sonnenaktivität und der Ausbruch von großen Vulkanen einerseits, aber andererseits auch die durch industrielle Prozesse veränderte chemische Zusammensetzung der Atmosphäre. 
CCMs zeigen in konsistenter Weise, dass sich die Ozonschicht zurückbilden wird. ${ }^{[20,23]}$ Dies ist ein unmittelbarer Erfolg des Montreal-Protokolls und der sich daran anschlieBenden Nachfolgevereinbarungen. Numerische Simulationen mit CCMs belegen, dass sich die Erholung der Ozonschicht in einigen Regionen schneller vollziehen wird als in anderen Regionen, wo es wohlmöglich sogar zu einer Verzögerung der Regenerierung der Ozonschicht kommen wird. Es zeigt sich, dass die Stratosphäre bei weiter steigenden Treibhausgaskonzentrationen wie erwartet weiter abkühlen wird, was in der mittleren und oberen Stratosphäre zu einer schnelleren Erholung der Ozonschicht führt. In der polaren unteren Stratosphäre kommt es in den Frühlingsmonaten zu einer Verlangsamung bei der Rückbildung der Ozonschicht. Dort führen niedrigere Temperaturen zu einer stärkeren Bildung von PSCs. Diese Ergebnisse bestätigen, dass die Erholung der Ozonschicht regional unterschiedlich verlaufen wird und keine simple Umkehrung des Abbaus in früheren Jahren bedeutet.

Eine vollständige Erholung der Ozonschicht auch unter Einschluss der Polarregionen wird etwa zur Mitte des Jahrhunderts erwartet. Wegen des Klimawandels scheint es möglich, dass ab Mitte dieses Jahrhunderts die Ozonschichtdicke über die in früheren Jahren gemessene Dicke hinaus zunimmt (Abbildung 7). Die Ergebnisse von vielen numerischen Simulationen mit verschiedenen gekoppelten CCMs sind hierzu in ihrer Aussage eindeutig, wenn auch der Zeitpunkt dieser „Übererholung“ der Ozonschicht von den Atmosphärenmodellen unterschiedlich vorhergesagt wird. ${ }^{[23]}$ So zeigen z.B. die Ergebnisse des CCM E39C-A des Deutschen Zentrums für Luft- und Raumfahrt (DLR), dass die mit dem Klimawandel in Verbindung stehenden Vorgänge $\mathrm{zu}$ einer insgesamt beschleunigten Erholung der Ozonschicht führen. ${ }^{[24]}$ Bereits vor der Mitte dieses Jahrhunderts werden wieder Ozonwerte vorhergesagt, wie sie in den 1960er Jahren

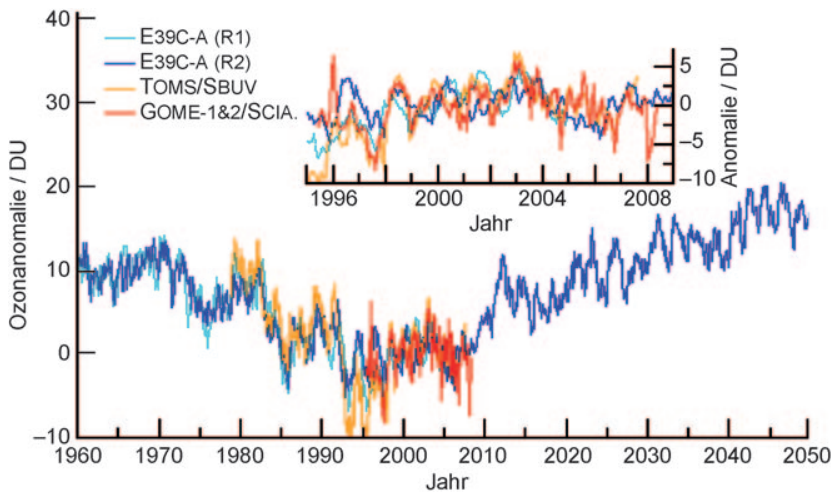

Abbildung 7. Gemittelte Anomalien der Ozongesamtsäule (in DU) für den Bereich von $60^{\circ} \mathrm{N}$ bis $60^{\circ} \mathrm{S}$. Es wurde jeweils der mittlere Jahresgang für den Zeitraum von 1995 bis 2004 abgezogen. Die orangefarbenen und roten Kurven stellen Messdaten dar, die von Satelliten-gestützten Instrumenten gewonnen wurden (TOMS/SBUV, GOME-1\&2/ SCIAMACHY). Die blauen Kurven zeigen Ergebnisse von numerischen Simulationen mit einem Klima-Chemie-Modell (E39C-A). Deutlich zu erkennen ist der Rückgang der stratosphärischen Ozonschicht in den 1980er und 1990er Jahren sowie der prognostizierte Anstieg bis Mitte dieses Jahrhunderts. Der Einschub zeigt einen Teil der Datenreihen in vergrößerter Form. (Neuausgabe der Abbildung 9 in [24].) gemessen wurden. Zum Ende der Modellsimulation (20402050) zeigt sich deutlich, dass die Ozonschichtdicke größer ist als im Zeitraum 1960-1970, also in jener Zeit, in der der durch FCKWs verursachte, massive stratosphärische Ozonabbau noch keine offensichtliche Bedeutung hatte. Abbildung 7 zeigt aber auch, dass natürliche Vorgänge wie der 11-jährige Sonnenaktivitätszyklus und große Vulkanausbrüche (Agung 1963, El Chichon 1982, Pinatubo 1991) zu einer deutlichen Veränderung der Dicke der stratosphärischen Ozonschicht führen. Die gute Übereinstimmung zwischen den aus Beobachtungen abgeleiteten Veränderungen der Ozonschichtdicke und entsprechenden Modellrechnungen belegt die Qualität der numerischen Simulationen (siehe auch Abbildung 3). Das hier verwendete Modellsystem berücksichtigt offensichtlich die wichtigsten Einflussfaktoren auf die stratosphärische Ozonschicht. Deshalb sind Aussagen über die zukünftige Entwicklung der Ozonschicht im Rahmen der genannten Unsicherheiten belastbar.

\subsection{Unsicherheiten prognostischer Studien}

Zum Abschluss dieses Abschnitts sei noch auf weitere Unsicherheiten hinsichtlich der weiteren Entwicklung der stratosphärischen Ozonschicht und des Klimas hingewiesen.

So ist z.B. derzeit die zukünftige Entwicklung stratosphärischer Wasserdampfkonzentrationen mit sehr großen Unsicherheiten behaftet. ${ }^{[3,20]}$ Nach vorliegenden Modellstudien würde bei steigenden Troposphärentemperaturen der troposphärische Wasserdampfgehalt zunehmen und damit auch der Eintrag von Wasserdampf aus der Troposphäre in die tropische untere Stratosphäre. Abschätzungen auf der Grundlage numerischer Studien lassen darauf schließen, dass höhere Wasserdampfkonzentrationen den Anteil von Wasserstoffoxiden $\left(\mathrm{HO}_{x}\right)$ in der Stratosphäre erhöhen, die ihrerseits wieder die Ozonzerstörung beeinflussen [katalytischer Reaktionszyklus (5)/(6)]. Höhere Wasserdampfkonzentrationen würden in den Polarregionen während der Wintermonate $\mathrm{zu}$ einem erhöhten PSC-Bildungspotenzial führen und damit auch auf die Ozonchemie einwirken. Da Wasserdampf ein sehr wichtiges Treibhausgas ist, hätten Veränderungen der stratosphärischen Wasserdampfkonzentration auch Auswirkungen auf den Strahlungshaushalt der Erdatmosphäre.

Die stratosphärischen Wasserdampfkonzentrationen würden aber auch bei ansteigender Methankonzentration zunehmen (Methanoxidation: $\mathrm{CH}_{4}+2 \mathrm{O}_{2} \rightarrow \mathrm{CO}_{2}+2 \mathrm{H}_{2} \mathrm{O}$ ), was erwartungsgemäß die Ozonproduktion in der unteren Stratosphäre erhöhen würde. Auf der anderen Seite würden höhere Methankonzentrationen reaktives Chlor in der Atmosphäre binden [Gl. (20)]. Methan ist aber auch ein relevantes Klimagas, das im Rahmen des Kyoto-Protokolls überwacht wird. Höhere atmosphärische Methankonzentrationen hätten also Konsequenzen für das Klima und die Chemie der Atmosphäre. Etwa ein Drittel der Emissionen von Methan kommt aus Feuchtgebieten, Wäldern, von Vegetationsbränden, aus Ozeanen und anderen natürlichen Quellen. Der Rest stammt aus der Produktion und Weiterarbeitung von Öl und Erdgas, von Viehherden, Abfalldepo- 
nien und anderem menschlichem Handeln. Ein starker Anstieg der Methanemission wird unter anderem erwartet, wenn Permafrost-Böden zunehmend auftauen.

Ein weiterer Anstieg der atmosphärischen Konzentrationen von Lachgas würde die Menge der stratosphärischen Stickoxide $\left(\mathrm{NO}_{x}\right)$ erhöhen, was den Ozongehalt in der mittleren und oberen Stratosphäre verringern würde [katalytischer Reaktionszyklus (5)/(6)]. Lachgas entstammt sowohl natürlichen (z.B. Ozeanen, tropischen Wäldern) als auch anthropogenen Quellen (z.B. Emissionen aus kultivierten Böden, bei industriellen Prozessen, bei der Verbrennung fossiler Treibstoffe, Biomasse und Biokraftstoffe) $.^{[28]} \mathrm{N}_{2} \mathrm{O}-$ Emissionen nahe der Erdoberfläche sind die wichtigste Quelle für Stickoxide $\left(\mathrm{NO}_{x}\right)$ in der Stratosphäre, obwohl nur etwa $10 \%$ des emittierten $\mathrm{N}_{2} \mathrm{O}$ in $\mathrm{NO}_{x}$ umgewandelt werden. $\mathrm{N}_{2} \mathrm{O}$ ist auch ein wichtiges Treibhausgas und wird daher durch das Kyoto-Protokoll überwacht. Bisher wurde Lachgas im Zusammenhang mit dem Montreal-Protokoll nicht als Ozon zerstörende Substanz bewertet und reguliert, obwohl die Emissionen von Lachgas, verglichen mit den Emissionen aller anderen Ozon zerstörenden Substanzen, heute am größten sind und es voraussichtlich auch für den Rest des 21. Jahrhunderts bleiben werden. $\mathrm{N}_{2} \mathrm{O}$ könnte sich für den Rest dieses Jahrhunderts zum wichtigsten Quellgas hinsichtlich der Zerstörung der Ozonschicht entwickeln. ${ }^{[29]}$ Anhand dieses Beispiels wird nochmals deutlich, in welch enger Beziehung die Problematik des Klimawandels und die der Veränderung der stratosphärischen Ozonschicht stehen: Eine Regulierung des Lachgasanteils in der Erdatmosphäre ist nicht nur für den Schutz des Erdklimas von Bedeutung, sondern auch für die weitere Entwicklung der stratosphärischen Ozonschicht. Eine Reduzierung der $\mathrm{N}_{2} \mathrm{O}$-Emissionen würde sowohl den anthropogenen Treibhauseffekt mindern als auch die Erholung der Ozonschicht positiv beeinflussen.

Als letztes soll noch erwähnt werden, dass Änderungen der Emissionen sowohl von $\mathrm{NO}_{x}$ als auch von Nicht-MethanKohlenwasserstoffen troposphärische Konzentrationen des Hydroxylradikals $\left(\mathrm{OH}^{\cdot}\right)$ verändern und somit die Lebenszeiten und Konzentrationen von stratosphärischen Spurengasen wie Methan beeinflussen.

\section{Zusammenfassung}

Es ist offensichtlich, dass Erklärungen zur Variabilität der stratosphärischen Ozonschicht sehr komplex sind und dass Abschätzungen zur zukünftigen Entwicklung nicht so einfach sind, wie es auf den ersten Eindruck erscheinen mag. Einerseits wird die stratosphärische Ozonkonzentration durch natürliche Einflüsse wie die Variabilität der Sonneneinstrahlung oder Partikelemissionen durch große Vulkanausbrüche verändert. Ferner beeinflusst die interne Variabilität der Stratosphärenzirkulation die thermische Struktur der Stratosphäre und den Luftmassentransport. Die chemische Produktion und der chemische Abbau von Ozon werden durch photochemische Prozesse sowie homogene Gasphasenreaktionen und heterogene Chemie auf der Oberfläche von Partikeln (Aerosole, PSCs) bestimmt. Dabei muss beachtet werden, dass die chemische Zerstörung von Ozon in der
Gegenwart von PSCs (oder auch vulkanischen Aerosolen) von nichtlinearer Beschaffenheit ist. Andererseits wird das Verständnis atmosphärischer Abläufe und der Zusammenhänge zwischen den verschiedenen Prozessen noch dadurch erschwert, dass sich die atmosphärischen Bedingungen wegen erhöhter Treibhausgaskonzentrationen langfristig verändern. Der Klimawandel beeinflusst die Nettoproduktion von Ozon (darunter versteht man die Summe aus Ozonzerstörung und Ozonproduktion) sowohl auf direkte als auch auf indirekte Weise und bestimmt deshalb die Rate der stratosphärischen Ozonrückbildung, die in unterschiedlichen Höhen und geographischen Breiten verschieden sein wird. Die Abkühlung der Stratosphäre durch erhöhte Treibhausgaskonzentrationen hat in der oberen und der unteren (polaren) Stratosphäre entgegengesetzte Effekte: Einerseits reduzieren sich die Ozonabbauraten aus der Gasphasenchemie, andererseits erhöhen sich die Ozonabbauraten durch heterogene Reaktionen auf PSC-Teilchen. Dies hat eine beschleunigte Wiederherstellung der Ozonmengen in der oberen Stratosphäre und eine verlangsamte Rückbildung von Ozon im Bereich der unteren Stratosphäre zur Folge.

Darüber hinaus haben Änderungen der stratosphärischen Zirkulation das Potenzial, die Entwicklung der Ozonschicht im 21. Jahrhundert zu modifizieren. So könnte eine verstärkte Brewer-Dobson-Zirkulation in einer Atmosphäre mit erhöhten Treibhausgaskonzentrationen z. B. auch dazu führen, dass sich die stratosphärischen Windsysteme in den Wintermonaten dahingehend verändern, dass sich die zonalen Windgeschwindigkeiten reduzieren; in der Folge hätte man im Mittel höhere Stratosphärentemperaturen im Bereich der Polgebiete und deshalb weniger PSCs. Auf der Grundlage derzeit vorliegender numerischer Modellstudien zur Abschätzung der zukünftigen Entwicklung des Klimas wird davon ausgegangen, dass sich die Brewer-Dobson-Zirkulation bei weiter steigenden Treibhausgaskonzentrationen verstärken wird. ${ }^{[25-27]}$ Die Abschätzungen sind allerdings noch mit einigen Unsicherheiten behaftet. Zwar sind die atmosphärischen Prozesse, die für die Brewer-Dobson-Zirkulation verantwortlich sind, recht gut verstanden, jedoch ist der Einfluss des Klimawandels auf all diese Prozesse sowie deren Wechselwirkungen noch nicht in allen Details nachvollziehbar.

Anhand der dargelegten Zusammenhänge wird deutlich, dass es bei der Bewertung von Veränderungen in der Erdatmosphäre nicht damit getan ist, Vorgänge singulär zu betrachten. Veränderungen des Klimas und der chemischen Zusammensetzung der Atmosphäre sind eng miteinander verknüpft. Die internationalen Vereinbarungen zum Schutz der Ozonschicht (Montreal-Protokoll) haben eine Katastrophe verhindert. ${ }^{[30,31]}$ Darüber hinaus haben diese Vereinbarungen bereits einen sehr wichtigen Beitrag zur Eindämmung des Klimawandels geleistet, da FCKWs auch relevante Treibhausgase sind. In diesem Zusammenhang sollte man die immer noch ausstehenden Nachfolgevereinbarungen zum Kyoto-Protokoll (Klimaschutzabkommen) auch als wichtigen Beitrag zum Schutz der stratosphärischen Ozonschicht sehen. Dynamische, physikalische und chemische Prozesse in der Atmosphäre beeinflussen sich gegenseitig, zum Teil auf sehr komplexe Weise. Überraschende Entwicklungen können 
daher auch in Zukunft nicht ausgeschlossen werden. Wegen der vielen Einflussfaktoren sowie der komplexen Wechselwirkungen ist eine verlässliche Vorhersage zukünftiger Entwicklungen des Erdklimas und der stratosphärischen Ozonschicht für die Wissenschaft nach wie vor eine große Herausforderung.

Eingegangen am 16. März 2010

Online veröffentlicht am $\mathbf{0}$. 2010

[1] IPCC (Intergovernmental Panel on Climate Change), Climate Change 2007: The Physical Basis, Cambridge University Press, Cambridge and New York, 2007, S. 996.

[2] W. J. Randel, K. P. Shine, J. Austin, J. Barnett, C. Claud, N. P. Gillett, P. Keckhut, U. Langematz, R. Lin, C. Long, C. Mears, A. Miller, J. Nash, D. J. Seidel, D. W. J. Thompson, F. Wu, S. Yoden, J. Geophys. Res. 2009, 114, D02107.

[3] W. J. Randel, A Festschrift to Celebrate Alan Plumb's 60th Birthday, im Druck.

[4] D. W. J. Thompson, S. Solomon, J. Clim. 2009, 22, 1934-1943.

[5] V. Ramaswamy, M. D. Schwarzkopf, W. J. Randel, B. D. Santer, B. J. Soden, G. L. Stenchikov, Science 2006, 311, 1138-1141.

[6] M. Dall'Amico, L. J. Gray, K. H. Rosenlof, A. A. Scaife, K. P. Shine, P. A. Stott, Clim. Dynam. 2009, 34, 381-398.

[7] J. C. Farman, B. G. Gardiner, J. D. Shanklin, Nature 1985, 315, 207-210.

[8] S. Solomon, Rev. Geophys. 1999, 37, 275-316.

[9] S. Chapman, Mem. Roy. Meteor. Soc. 1930, 3, 103-125.

[10] D. R. Bates, M. Nicolet, J. Geophys. Res. 1950, 55, 301-327.

[11] P. J. Crutzen, J. Geophys. Res. 1971, 76, 7311-7327.

[12] H. S. Johnston, Science 1971, 173, 517-522.

[13] M. J. Molina, F. S. Rowland, Nature 1974, 249, 810-812.

[14] S. C. Wofsy, M. B. Mc Elroy, Y. L. Yung, Geophys. Res. Lett. 1975, 2, 215-218.

[15] S. Solomon, R. R. Garcia, F. S. Rowland, D. J. Wuebbles, Nature 1986, 321, 755-758.

[16] L. T. Molina, M. J. Molina, J. Phys. Chem. 1987, 91, 433-436.

[17] J. Haigh, J. Pyle, Q. J. Roy. Meteorol. Soc. 1982, 108, 551-574.

[18] A. W. Brewer, Q. J. Roy. Meteorol. Soc. 1949, 75, 351-363.

[19] G. M. B. Dobson, Proc. R. Soc. A 1956, 236, 187-193.

[20] WMO (World Meteorological Organization), Global Ozone Research and Monitoring Project, Genf (Schweiz), 2007, Report No. 50 , S. 572.

[21] J. R. Holton, P. H. Haynes, M. E. McIntyre, A. R. Douglass, R. B. Rood, L. Pfister, Rev. Geophys. 1995, 33, 403-439.

[22] A. Stenke, M. Dameris, V. Grewe, H. Garny, Atmos. Chem. Phys. 2009, 9, 5489-5504.
[23] SPARC CCMVal Report, Report on the Evaluation of Chemistry Climate Models 2010, SPARC-Report No. 5, WCRP-132, WMO/TD-No. 1526.

[24] D. G. Loyola, R. M. Coldewey-Egbers, M. Dameris, H. Garny, A. Stenke, M. van Roozendael, C. Lerots, D. Balis, M. Koukouli, Int. J. Remote Sens. 2009, 30, 4295-4318.

[25] N. Butchart, A. A. Scaife, M. Bourqui, J. de Grandpre, S. H. E. Hare, J. Kettleborough, U. Langematz, E. Manzini, F. Sassi, K. Shibata, D. Shindell, M. Sigmond, Clim. Dynam. 2006, 27, 727 741.

[26] N. Butchart, I. Cionni, V. Eyring, T. G. Shepherd, D. W. Waugh, H. Akiyoshi, J. Austin, C. Brühl, M. P. Chipperfield, E. Cordero, M. Dameris, R. Deckert, S. Dhomse, S. M. Frith, R. R. Garcia, A. Gettelman, M. A. Giorgetta, D. E. Kinnison, F. Li, E. Mancini, C. McLandress, S. Pawson, G. Pitari, D. A. Plummer, E. Rozanov, F. Sassi, J. F. Scinocca, K. Shibata, B. Steil, W. Tian, J. Climate 2010, DOI: 10.1175/2010JCLI3404.1.

[27] V. Eyring, D. W. Waugh, G. E. Bodeker, E. Cordero, H. Akiyoshi, J. Austin, S. R. Beagley, B. A. Boville, P. Braesicke, C. Brühl, N. Butchart, M. P. Chipperfield, M. Dameris, R. Deckert, M. Deushi, S. M. Frith, R. R. Garcia, A. Gettelman, M. A. Giorgetta, D. E. Kinnison, E. Mancini, E. Manzini, D. R. Marsh, S. Matthes, T. Nagashima, P. A. Newman, J. E. Nielsen, S. Pawson, G. Pitari, D. A. Plummer, E. Rozanov, M. Schraner, J. F. Scinocca, K. Semeniuk, T. G. Shepherd, K. Shibata, B. Steil, R. S. Stolarski, W. Tian, M. Yoshiki, J. Geophys. Res. 2007, 112, D16303.

[28] IPCC (Intergovernmental Panel on Climate Change), Climate Change, Cambridge University Press, Cambridge and New York, 2001, S. 881.

[29] A. R. Ravishankara, J. S. Daniel, R. W. Portmann, Science 2009, 326, 123 -125; Highlight: M. Dameris, Angew. Chem. 2010, 122, 499-501; Angew. Chem. Int. Ed. 2010, 49, 489-491.

[30] O. Morgenstern, P. Braesicke, M. M. Hurwitz, F. M. O'Connor, A. C. Bushell, C. E. Johnson, J. A. Pyle, Geophys. Res. Lett. 2008, 35, L16811.

[31] P. A. Newman, L. D. Oman, A. R. Douglass, E. L. Fleming, S. M. Frith, M. M. Hurwitz, S. R. Kawa, C. H. Jackman, N. A. Krotkov, E. R. Nash, J. E. Nielsen, S. Pawson, R. S. Stolarski, G. J. M. Velders, Atmos. Chem. Phys. 2009, 9, 2113-2128.

[32] C. von Savigny, C. S. Haley, C. E. Sioris, I. C. McDade, E. J. Llewellyn, D. Degenstein, W. F. J. Evans, R. L. Gattinger, E. Griffioen, E. Kyrölä, N. D. Lloyd, J. C. McConnell, C. A. McLinden, G. Megie, D. P. Murtagh, B. Solheim, K. Strong, Geophys. Res. Lett. 2003, 30, 1755.

[33] T. A. Shaw, T. G. Shepherd, Nat. Geosci. 2008, 1, 12-13.

[34] P. J. Crutzen, Angew. Chem. 1996, 108, 1878-1898; Angew. Chem. Int. Ed. 1996, 35, 1758-1777; M. J. Molina, Angew. Chem. 1996, 108, 1900-1907; Angew. Chem. Int. Ed. 1996, 35, 17781785; F. S. Rowland, Angew. Chem. 1996, 108, 1908-1921; Angew. Chem. Int. Ed. 1996, 35, 1786-1798. 


\section{Aufsätze}

Ozonschicht

M. Dameris*

IIII-IIII

Klimawandel und die Chemie der Atmosphäre - wie wird sich die stratosphärische Ozonschicht entwickeln?

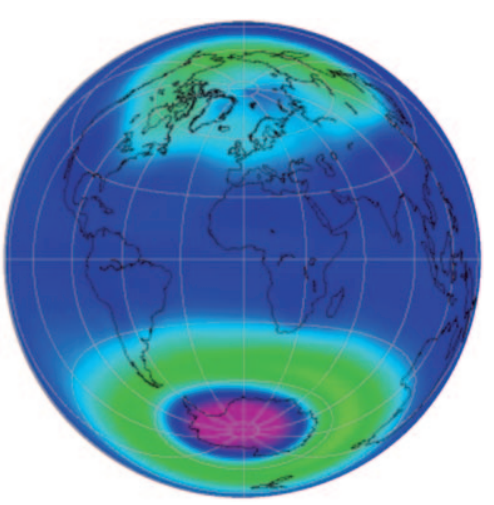

Seit Beginn der Industrialisierung vor ca. 150 Jahren ist die Konzentration von Treibhausgasen wie $\mathrm{CO}_{2}$ in der Erdatmosphäre deutlich gestiegen, mit entsprechenden Auswirkungen auf das Erdklima. Parallel beobachtet man seit mehr als 25 Jahren eine Zerstörung der Ozonschicht (siehe z. B. lila Bereiche des Globus - das Ozonloch), die durch die Emission von Fluorchlorkohlenwasserstoffen verursacht ist. Die künftige Entwicklung der Ozonschicht und des Klimas stehen in enger Beziehung. 